\title{
Sex Differences in Heat Shock Protein 72 Expression in Peripheral Blood Mononuclear Cells to Acute Exercise in the Heat
}

\author{
Trevor Gillum ${ }^{1,{ }^{*},}$ Matthew Kuennen ${ }^{2}$, Cheryl Gourley ${ }^{3}$, Karol Dokladny $^{4}$, Suzanne Schneider ${ }^{3}$, \\ Pope Moseley ${ }^{4}$ \\ 1 Department of Kinesiology, California Baptist University, Riverside, USA \\ 2 Department of Sports and Exercise Science, West Texas A\&M University, Canyon, USA \\ ${ }_{4}^{3}$ Department of Health, Exercise and Sport Sciences, The University of New Mexico, Albuquerque, USA \\ 4 Department of Internal Medicine, The University of New Mexico, Albuquerque, USA \\ ${ }^{*}$ Corresponding author: Trevor L. Gillum, Kinesiology Department, California Baptist University, 8432 Magnolia Ave, Riverside, CA 92504. Tel: +1-9513434950, Fax: +1-9513434343, E- \\ mail: tgillum@calbaptist.edu. \\ Received: October 26, 2012; Revised: March 17, 2013; Accepted: April 27, 2013
}

\begin{abstract}
Background: Heat shock protein 72 (Hsp72) is responsible for maintaining critical cellular function during heat stress. Hsp72 confers thermotolerance and may play a role in heat acclimation. Animal research suggests a difference between sexes in Hsp72 expression in response to exercise, however, human data is lacking.

Objectives: To determine sex differences in intracellular heat shock protein 72 (Hsp72) following exercise in the heat.

Patients and Methods: Nine non-heat acclimated women with normal menstrual cycles (VO2pk $\left.58 \pm 5 \mathrm{~mL} \cdot \mathrm{kgFFM}^{-1} \cdot \mathrm{min}^{-1}\right)$ and nine nonheat acclimated men $\left(\mathrm{VO} 2 \mathrm{pk} 60 \pm 7 \mathrm{ml} \cdot \mathrm{kgFFM}^{-1} \cdot \mathrm{min}^{-1}\right)$ completed 2 treadmill bouts at $60 \% \mathrm{VO} 2 \mathrm{pk}$ for 60 min in a $42^{\circ} \mathrm{C}, 20 \% \mathrm{RH}$ environment. Women were tested in follicular (fol) and luteal (lut) phases. The duplicate trials were separated by 12 days for men and women. Blood samples were drawn pre, immediately post, 1 , and $4 \mathrm{hrs}$ post-exercise.

Results: Men and women differed in their Hsp72 response after exercise (timeX sex X trial interaction; $\mathrm{P}<0.05)$. Men increased Hsp72 after exercise more than women. Both men and women produced less Hsp72 during trial 2 compared to trial 1 . Estrogen $(r=0.24 ; \mathrm{P}>0.05)$ and progesterone $(r=0.27, \mathrm{P}>0.05)$ concentrations were not correlated with $\mathrm{Hsp72}$.

Conclusion: Our findings suggest that men and women differ in their cellular stress response. Men up-regulated Hsp72 after a single bout of exercise in the heat, which persists for 12 days, suggesting an accumulation of Hsp72 which may lead to acquired cellular thermotolerance.
\end{abstract}

Keywords: Menstrual Phase; Thermoregulation; Endogenous antioxidants; Thermotolerance

\section{Background}

The cellular stress response is characterized by the accumulation of intracellular heat shock proteins, including the highly heat inducible $72-\mathrm{kDa}$ heat shock protein 72 (Hsp72). Hsp72 expression is linked to thermotolerance (1) in that increased Hsp72 protects cells against normally lethal increases in core temperature $(2,3)$. Hsp72 may protect cells by acting as an intracellular chaperone enhancing protein folding (4), inhibiting intracellular proinflammatory cytokine synthesis (5-8), and/or limiting cellular apoptosis (7). Males have shown an increased expression of Hsp72 in peripheral blood mononuclear cells (PBMC) $(6,7,9,10)$, skeletal muscle (11), brain (12) and liver (13) within 4 hrs after exercise or heat stress.

Given that Hsp72 is accumulated in response to stress, it is interesting that animal studies have shown a difference in the expression of Hsp72 between sexes. Under resting conditions, basal levels of Hsp72 in cardiac and renal tissue are higher in female compared to male rats $(14,15)$. However, in response to running $(16,17)$ and hyperthermia (18), male animals are able to express higher Hsp72 than females in the gastrocnemius and cardiac tissue. In the above studies, sham treated ovariectomized females had similar post stress Hsp72 levels as males, while estrogen treated ovariectomized females showed unaltered Hsp72 levels after stress, similar to intact females. Animal studies suggest an increased basal expression of Hsp72 may reduce the cellular response to an exercise stress and thus limit the need for additional Hsp72 production after the stress (19). The mechanism behind the relationship between estrogen and the blunted intracellular Hsp72 response to stress currently is not known. It was suggested

Implication for health policy/practice/research/medical education:

This is the first human study that has assessed intracellular Hsp72 in response to stress. These data raise interesting questions regarding thermotolerance, heat acclimation, and the cellular stress response between men and women.

Copyright (C) 2013, Research Institute For Endocrine Sciences and Iran Endocrine Society; Published by Kowsar. This is an Open Access article distributed under the terms of the Creative Commons Attribution License (http://creativecommons.org/licenses/by/3.0), which permits unrestricted use, distribution, and reproduction in any medium, provided the original work is properly cited. 
that estrogen mediates this effect through a nongenomic hormonal pathway (17). Currently, there are no human studies that have tested for possible sex differences in the cellular stress response to exercise in the heat.

\section{Objectives}

Therefore, the objective of this study was to compare the expression of Hsp72 in PBMC at rest and after exercise in the heat in men and women. We hypothesized that women would have an increased baseline expression of Hsp72, but an attenuated post exercise response compared to men.

Furthermore, we examined if women in follicular (fol) and luteal (lut) phases of the menstrual cycle differed in their amount of Hsp72 expressed in response to exercise in the heat. We hypothesized that during lut, when estrogen levels are elevated, there would be a decrease in Hsp72 at rest and after exercise compared to fol.

\section{Materials and Methods}

\subsection{Subjects}

Eighteen (9 men and 9 women) subjects completed two treadmill sessions in a hot, dry environment (42.3 $\pm 1^{\circ} \mathrm{C}, 22.5 \pm 12 \%$ relative humidity). Men were matched with women for aerobic fitness ( $\mathrm{ml} \cdot \mathrm{kg}$ lean body mass ${ }^{-1}$ $\min ^{-1}$ ) and age (Table 1). None of the women were taking hormonal contraceptives and all had menstrual cycles of 28-32 days for three months prior to testing. Women were tested during the fol (day $7 \pm 2$ ) and lut (days $20 \pm$ 1) phases of their menstrual cycle. Progesterone concentration was used to validate the presence of the luteal phase, as previously done $(20,21)$. The same length of time elapsed between the two exercise trials for men and women (M: $12 \pm 4$ days, W: $12 \pm 2$ days). Each exercise trial for a given subject was conducted at the same time of day. Between exercise trials, subjects were asked to continue their normal physical activity patterns. The University of New Mexico's Institutional Review Board approved this protocol and the subjects provided written, informed consent prior to participation.

\subsection{Preliminary Testing}

Body composition and cardiorespiratory fitness were assessed for all subjects. Three site skinfold (Lange, Beta Technology, Santa Cruz, CA) measurements (M: chest, abdomen, thigh; W: triceps, suprailiac, thigh) were used to determine percent body fat. Each site was measured in triplicate and the mean value was used to calculate percent body fat (22). A continuous graded treadmill test in a temperate room $\left(22-24^{\circ} \mathrm{C}, 30 \% \mathrm{RH}\right)$ was used to determine VO2peak. VO2peak was assessed through open circuit spirometry (ParvoMedics, Sandy, UT) and defined as the highest 30 second value when 2 of the following criteria were met: 1) a plateau in $\mathrm{VO} 2$ (change in $\mathrm{VO} 2<150 \mathrm{~mL}$ $\mathrm{min}^{-1}$ ) with increased workload, 2) a maximal respiratory exchange ratio greater than 1.1 and 3) heart rate greater than $95 \%$ of the age predicted maximum (220-age). VO2 peak was expressed per $\mathrm{mL}$ of fat free mass (FFM) so that the aerobic fitness of sexes could be compared.

\subsection{Experimental Design}

Each subject performed two exercise trials (treadmill running at $60 \% \mathrm{VO} 2$ peak for $60 \mathrm{~min}$ ) in an environmental chamber maintained at $42^{\circ} \mathrm{C}, 20 \% \mathrm{RH}$. The trials for women were counterbalanced so that 4 subjects performed the first exercise bout in the lut phase while 5 subjects performed the first exercise bout in the fol phase. Plasma estrogen and progesterone values were obtained to corroborate the appropriate menstrual cycle phase.

\subsection{Exercise Trial}

Data collection took place during the Fall and Winter months (October - February) to limit the effects of heat acclimation. Subjects were instructed to avoid exercise and alcohol for $24 \mathrm{hrs}$ and to avoid caffeine for $12 \mathrm{hrs}$ prior to each exercise trial. Subjects were given a list of high carbohydrate foods to consume for dinner on the night before and for breakfast on the morning before each trial. Subjects were asked to consume the same foods before the two trials.

On the day of the trial, nude body weight was recorded to the nearest $0.1 \mathrm{~kg}$ (Seca Scale, Birmingham, UK) and urine osmolality was used to assess the subjects' hydration status (Advanced Osmometer, Model 303, Advanced Instruments Inc, Norwood, MA).

Core temperature (Tr) was measured by inserting a thermistor (YSI precision 4400 Series, Yellow Springs Inc, Yellow Springs, $\mathrm{OH}) 10 \mathrm{~cm}$ past the anal sphincter. An intravenous catheter was inserted in an antecubital vein and kept patent by infusing $3 \mathrm{ml}$ of isotonic saline every 15 min during and after the exercise trial. Samples were drawn pre, immediately post, $1 \mathrm{hr}$ post and $4 \mathrm{hrs}$ post exercise.

The exercise intensity was set to elicit $60 \%$ of VO2peak. The intensity of exercise was selected such that active individuals who were not endurance trained would be able to complete the protocol.VO2 was measured every 15 min during exercise. The speed and grade were adjusted during the first 15 min of trial 1 to obtain a $\mathrm{VO} 2$ of $60 \%$ of VO2peak. This exercise level was maintained for the remainder of the exercise trial. When subjects repeated the exercise trial, the treadmill speed and grade were identical to the first exercise trial. During the first exercise trial, the subjects were allowed to drink water ad libitum. Each subject then ingested the same volume of water during their second trial. After exercise, each subject dried themselves with a towel and obtained their nude body weight. Sweat loss was calculated as the difference in pre to post 
weight, corrected for fluid intake. One male and one female subject were unable to complete their first $60 \mathrm{~min}$ exercise trial due to nausea and light headedness. When the trial was repeated, these subjects then exercised for this same duration. Hsp72 for these subjects was not anomalously higher after the first trial compared with the second. Therefore, data from these subjects are included in the analysis.

\subsection{Blood Collection and Preparation}

Three $\mathrm{ml}$ of blood were drawn at each time point and transferred to EDTA treated tubes. Half of the blood was aliquotted to measure hematocrit, plasma estrogen, and progesterone. The other half was used to measure Hsp72. Hematocrit was analyzed within 15 min of the blood draw and used to correct plasma protein concentrations for plasma volume changes during exercise as described elsewhere (23). After measuring hematocrit (microcentrifuge technique), the blood was centrifuged and the plasma separated and stored at $-80^{\circ} \mathrm{C}$ until later analysis. PBMC's were separated from $1 \mathrm{~mL}$ of blood using density gradient centrifugation (15 min, 2100 RPM, O ACC) with $1.077 \mathrm{~g} / \mathrm{mL}$ Histopaque (Sigma-Aldrich, St. Louis, MO). PBMC were washed with phosphate buffered saline
(PBS) and then treated with Reagent A (Fix and Perm kit, Invitrogen, Carlsbad, CA) and incubated at room temperature for $15 \mathrm{~min}$. Cells were then washed and treated with Reagent B (Fix and Perm kit, Invitrogen, Carlsbad, CA) combined with a monoclonal Hsp72 FITC antibody (Assay Designs, Ann Arbor, MI) at $100 \mu \mathrm{g} / \mathrm{mL}$. Cells were incubated for 20 minutes in the dark at room temperature. Cells were washed a final time, and then diluted in 300 mL sheath fluid and analyzed using a FACSCAN cytometer (BD Scientific, San Jose, CA). Ten thousand events were collected. Data was analyzed using Cellquest software (BD Scientific, San Jose, CA). The amount of protein produced per cell population was quantified as mean fluorescent intensity (MFI). To determine MFI, cells were gated and corrected for auto-fluorescence based upon unstained control. The gating strategy is shown in Figure 1.

Plasma was analyzed for estrogen and progesterone using ELISA kits (Genway Bioscience, San Diego, CA) according to manufacturer's instruction. The minimum detectable concentration was $5 \pm 2 \mathrm{pg} / \mathrm{mL}$ for estradiol and 0.08 $\pm 0.03 \mathrm{ng} / \mathrm{mL}$ for progesterone. Inter-assay variability was $6 \%$ for estradiol and $8.8 \%$ for progesterone. Intra-assay variability was $4.6 \%$ for estradiol and $9.7 \%$ for progesterone.

Figure 1. Representative Flow Cytometry Data From PBMCs.
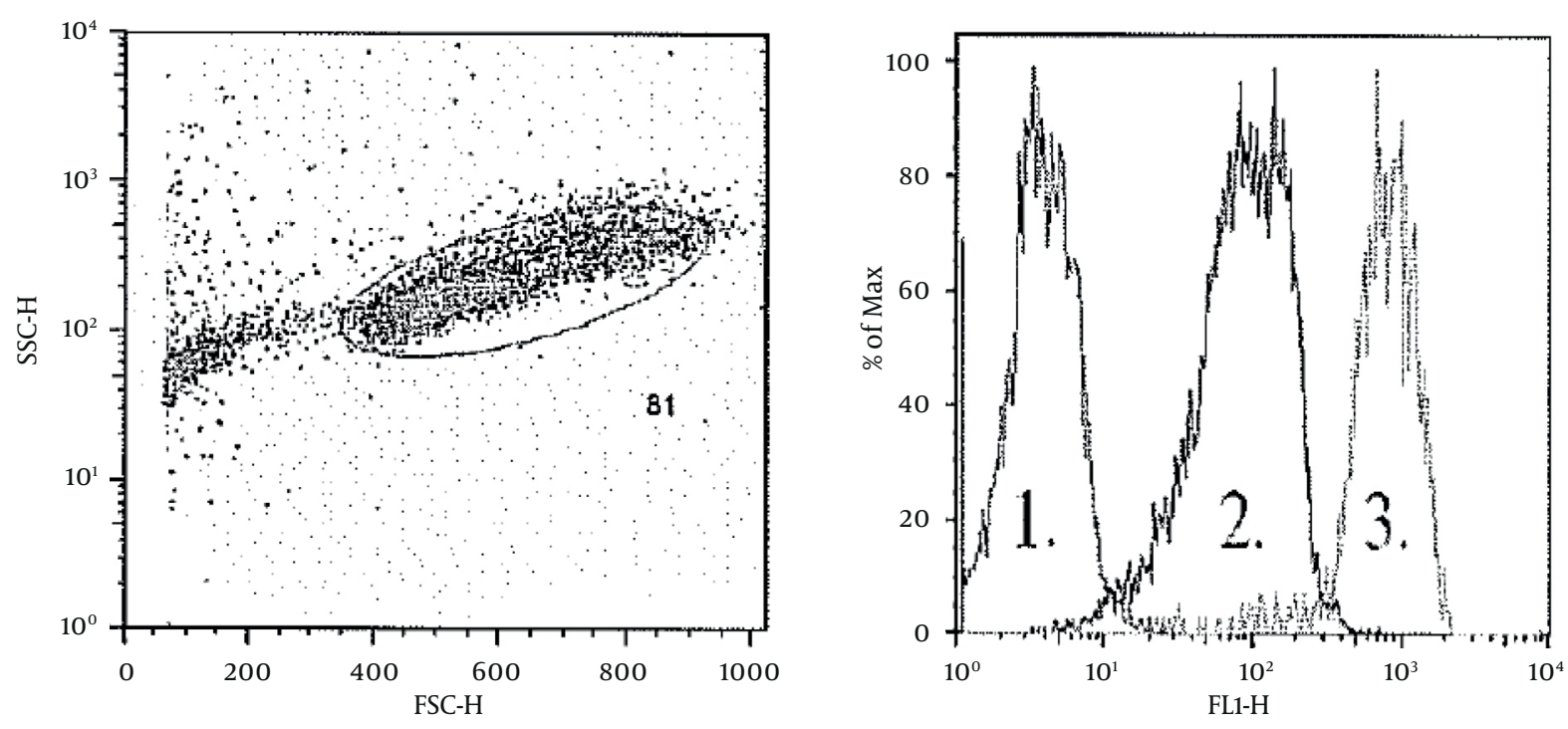

A) Gating strategy: SSC vs FSC for PBMC. B) Expression of Hsp72 in 1) unstained cells, 2) pre exercise, and 3 ) after $42^{\circ} \mathrm{C}$ incubation.

\subsection{Statistical Analysis}

Hsp72 was expressed as MFI, and, to more accurately assess sex differences due to exercise, pre exercise values were normalized to 1 . Thus, the post exercise expression of Hsp72 was expressed as the change from the pre exercise value as previously reported $(24,25)$. A three factor (time $\mathrm{x}$ trial $\mathrm{x}$ sex) ANOVA was used to determine changes due to exercise. Sampling times included pre-exercise, immediately post exercise, $1 \mathrm{hr}$ post exercise, and $4 \mathrm{hrs}$ post-exercise.

Menstrual cycle comparisons were done only for those women in whom their phase of the cycle was confirmed by hormonal analysis (see results). A two-factor (time $\mathrm{x}$ phase) repeated measures ANOVA was used to test for differences due to the menstrual cycle phase. Here we com- 
pared fol vs. lut data, $\mathrm{n}=7$. To determine the potential effect of ovarian hormones on Hsp72, a correlation analysis between estrogen and Hsp72 in addition to progesterone and Hsp72 was conducted for all women.

All descriptive data were analyzed using a t-test (weight, aerobic capacity, \% body fat, and hormonal analysis of estrogen and progesterone). Independent t-tests were used to compare men's and women's values for Tr, HR, urine osmolality, and relative exercise intensity.

The necessary $n$ size was estimated to be 8 subjects, given a power of 0.80 and an alpha level of 0.05 , using Statistica. Because sex differences in Hsp72 have not been assessed, we estimated $n$ size according to the change from baseline in monocyte's of males after treadmill exercise in the heat $(6,9)$.

Statistical significance was set at $\alpha \leq 0.05$ and analysis was performed using Statistica 10 (StatSoft Inc, Tulsa, OK).
Data were assessed for normality and homogeneity of variance prior to statistical analysis. Hsp72 data were not normally distributed and thus log transformed prior to analysis. Tukey's post hoc test was used if necessary. Data is presented as mean \pm SD in text and tables. For simplicity, data in figures is presented as mean \pm SEM .

\section{Results}

\subsection{Subject Characteristics}

Men and women differed significantly for height $(\mathrm{P}=$ $0.01)$, weight $(P=0.01)$ \% body fat $(P=0.01)$, and aerobic capacity expressed as $\mathrm{ml} \mathrm{kg}^{-1} \mathrm{~min}^{-1}(\mathrm{P}=0.03)$. However, when aerobic capacity was expressed relative to fat free mass, there was no difference between sexes (Table 1). For men, trial 2 was repeated $12 \pm 4$ days after trial 1 . For women, trial 2 was repeated $12 \pm 2$ days after trial 1 .

Table 1. Subject Characteristics.

\begin{tabular}{|c|c|c|c|c|c|c|}
\hline & Age, $y$ & Height, cm & Weight, kg & V02pl, $\mathrm{ml}^{-1} \mathrm{~kg}^{-1} \mathrm{~min}^{-1}$ & V02pk, $\mathrm{ml}^{-1} \mathrm{kgFFM}^{-1} \mathrm{min-1}$ & Body Fat, \% \\
\hline Men & $26 \pm 5$ & $182 \pm 7^{\mathrm{a}}$ & $81.0 \pm 15^{\mathrm{a}}$ & $52.6 \pm 8^{a}$ & $60.0 \pm 7.7$ & $12.0 \pm 5.5^{\mathrm{a}}$ \\
\hline Women & $24 \pm 3$ & $170 \pm 3$ & $63.1 \pm 12$ & $44.9 \pm 5$ & $58.8 \pm 5.2$ & $21.4 \pm 2.5$ \\
\hline
\end{tabular}

${ }^{\mathrm{a}}$ Difference $(\mathrm{P}<0.05)$ between sexes.

\subsection{Exercise Response}

Men and women did not differ in starting or ending $\mathrm{T}$ $r$, HR, pre or post urine osmolality, or relative exercise intensity. Men had significantly greater sweat rates than women $(P=0.01)$. One woman experienced heat illness symptoms at 45 min during trial 1, and one male subject stopped exercising for similar reasons at $30 \mathrm{~min}$ on trial
1. The second bout for both subjects was identical in duration and intensity to the first bout. Hsp72 levels for these subjects were not anomalously higher after the first trial compared with the second. Therefore, data from these subjects are included in the analysis. All other subjects completed 60 min of exercise. There was no significant effect of menstrual phase on baseline or exercise $\mathrm{T} r$, urine osmolality, ending HR, or exercise intensity (Table 2).

\begin{tabular}{|c|c|c|c|c|c|c|c|}
\hline & Pre Tc $(C)$ & End Tc $(C)$ & $\begin{array}{l}\text { Pre Urine } \\
\text { Osm, mOsm/ } \\
\text { kg }\end{array}$ & $\begin{array}{l}\text { Post Urine } \\
\text { Osm, mOsm/kg }\end{array}$ & End HR & $\begin{array}{l}\text { Mean \% V02pk, } \\
\text { mL/kg/min }\end{array}$ & $\begin{array}{l}\text { Sweat Rate, } \\
\text { mL/min }\end{array}$ \\
\hline $\begin{array}{l}\text { Women } \\
\text { Trial } 1\end{array}$ & $37.21 \pm 0.08$ & $39.05 \pm 0.15$ & $499 \pm 143$ & $453 \pm 105$ & $162 \pm 4$ & $60 \pm 1.3$ & $16 \pm 2$ \\
\hline $\begin{array}{l}\text { Women } \\
\text { Trial } 2\end{array}$ & $37.17 \pm 0.06$ & $38.95 \pm 0.17$ & 42482 & $362 \pm 62$ & $160 \pm 4$ & $58 \pm 1.6$ & $17 \pm 2$ \\
\hline Men Trial 1 & $37.04 \pm 0.07$ & $39.19 \pm 0.09$ & $559 \pm 72$ & $520 \pm 83$ & $166 \pm 2$ & $57 \pm 1.5$ & $29 \pm 2^{b}$ \\
\hline Men Trial 2 & $36.98 \pm 0.11$ & $39.07 \pm 0.14$ & $442 \pm 91$ & $547 \pm 95$ & $163 \pm 2$ & $58 \pm 0.75$ & $26 \pm 4^{b}$ \\
\hline
\end{tabular}

${ }^{\mathrm{a}}$ Response to $60 \mathrm{~min}$ of treadmill running at $60 \% \mathrm{VO} 2 \mathrm{pk}$ in a $42^{\circ} \mathrm{C}$ environment

$\mathrm{b}_{\mathrm{P}<0.05 \text { from Women's trials. }}$

\subsection{Menstrual Phase Hormones}

Data from two subjects were removed from the menstrual cycle comparison after examining their progesterone values. In these subjects, progesterone values were not higher in the lut compared to fol phase. Therefore, all menstrual phase analyses are shown with $n=7$. Of the seven subjects analyzed for menstrual phase differences, five completed their first exercise trial in the fol phase, and two subjects exercised in the lut phase first. Estrogen and progesterone levels were significantly higher $(\mathrm{P}=.01)$ in the lut compared to fol phase (Table 3). 
Gillum Tet al.

Table 3. Sex Hormones and Menstrual Cycle Phase $(n=7)($ mean \pm SD).

\begin{tabular}{lllllll}
\hline & Estrogen, pmol/L & Progesterone, nmol/L & Ending HR & \% VO2pk & Pre Tc(C) & End Tc(C) \\
\hline Follicular & $344 \pm 95^{\mathrm{a}}$ & $3.35 \pm 1.2^{\mathrm{a}}$ & $160 \pm 7.1$ & $56 \pm .002$ & $37.19 \pm 0.16$ & $39.01 \pm 0.53$ \\
Luteal & $436 \pm 89$ & $30.1 \pm 13.9$ & $163 \pm 7.0$ & $58 \pm 0.01$ & $37.35 \pm 0.28$ & $39.06 \pm 0.44$ \\
\hline
\end{tabular}

$\mathrm{a}_{\mathrm{P}}<0.05$ from Lut

\subsection{Hsp72 Exercise Response}

There was no difference in pre exercise values of $\mathrm{Hsp} 72$ MFI between sexes on trial 1 (men: 111 \pm 30 , women: $121 \pm$ 34 ) or 2 (men: $145 \pm 38$, women: $151 \pm 43$ ). The normalized, overall Hsp72 response after exercise was greater in men than women (time $\mathrm{x}$ trial $\mathrm{x}$ sex interaction $\mathrm{P}=0.01$ ) (Figure 2). Men expressed higher Hsp72 at +1 and +4 hrs post exercise compared to women pre and post during trial 1. However, this sex difference did not occur during trial 2. While the normalized increase in Hsp72 from pre exercise values was not significant in women during either trial, men increased $\mathrm{Hsp} 72$ at +1 and +4 hrs from pre exercise values in trial 1 (time $x$ sex interaction $P=0.05$ ). Both men and women expressed less Hsp72 in trial 2 compared to trial 1 (time $\mathrm{x}$ trial interaction $\mathrm{P}=<.001$ ).

Figure 2. Normalized Hsp72, Exercise Responses

Hsp72

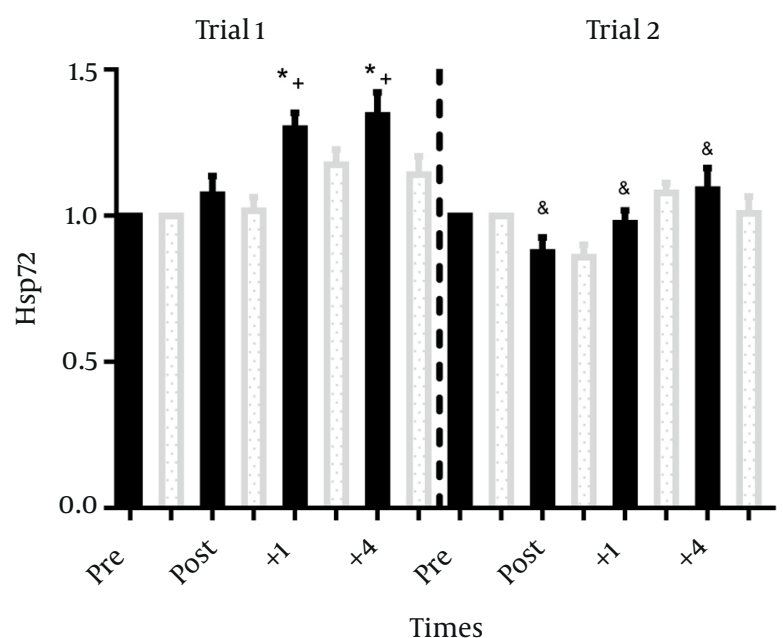

Hsp72 response to $60 \mathrm{~min}$ of treadmill running at $60 \% \mathrm{VO} 2 \mathrm{pk}$ in a $42^{\circ} \mathrm{C}$ environment, as a percentage of baseline values. Dark bars represent men, open bars represent women. The dashed line separates trial 1 from trial 2. There was a time $\mathrm{x}$ trial $\mathrm{x}$ sex interaction: ${ }^{*} \mathrm{P}<0.05$ from pre exercise values for men trial 1 . and $\mathrm{P}<0.05$ from trial 1 at the same time points for men. $+\mathrm{P}<0.05$ from women pre and post exercise during trial 1. Data represent mean \pm SEM.

\subsection{Hsp72 Exercise Response - Menstrual Phase}

In the 7 women included in the menstrual phase analysis, there was no difference in pre exercise values of Hsp72 MFI expression in fol (117 \pm 14$)$ versus lut $(123 \pm 24)$ phases.
The normalized, overall Hsp72 response increased after exercise (main effect of time, $\mathrm{P}=0.01$ ), but was not different between fol and lut phases (Figure 3). For all female subjects, estrogen $(r=0.24,0.12,0.17,-0.7)$ and progesterone $(r=0.27,0.13,0.14,-0.1)$ concentrations were not correlated to Hsp72 MFI values at pre, post, +1 , and +4 hrs post exercise, respectively (Figures 4 and 5).

Figure 3. Menstrual Phase

$$
\text { Menstrual Phase }
$$

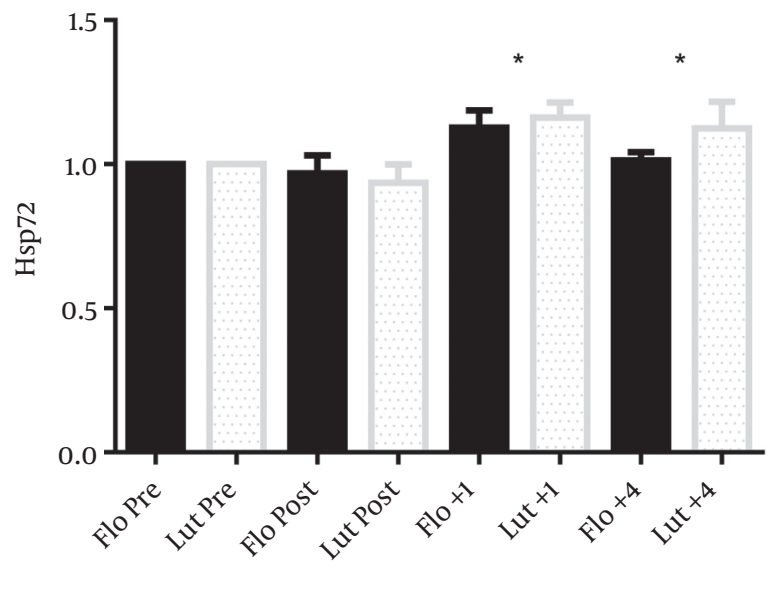

Hsp72 response to $60 \mathrm{~min}$ of treadmill running at $60 \% \mathrm{VO} 2 \mathrm{pk}$ in a $42^{\circ} \mathrm{C}$ environment, as a percentage of baseline values. Closed bars represent follicular phase, open bars represent luteal phase $(n=7)$. There was a main effect of time: ${ }^{*} \mathrm{P}<0.05$ from pre values. Data represents mean \pm SEM.

Figure 4. Correlation between Estrogen and Hsp72.

Correlation Hsp72 and Estrogen

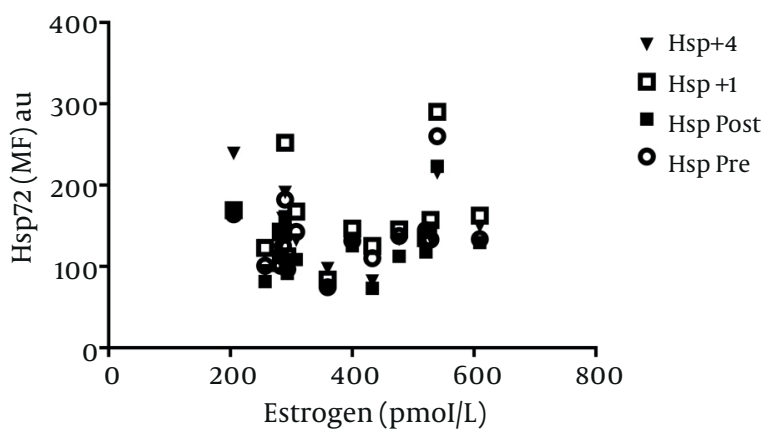

Pre exercise estrogen concentration was not correlated with Hsp72 at any time point. Data represents $n=9$. 
Figure 5. Correlation Between Progesterone and Hsp72.

Correlation Progesterone and Hsp72

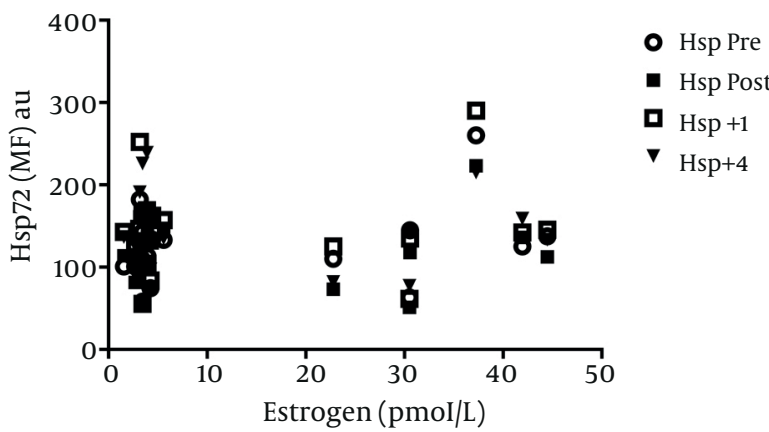

Pre exercise progesterone concentration was not correlated with Hsp72 at any time point. Data represents $n=9$.

\section{Discussion}

Our major finding is that men and women have different Hsp72 stress responses to exercise in the heat. Men expressed greater Hsp72 after exercise in trial 1 than women, and subsequently up-regulated their baseline Hsp72 expression by $\sim 30 \%$ before trial 2 . This increase in baseline Hsp72 led to reduced Hsp72 expression when the same exercise stress was repeated 12 days later. Women produced less Hsp72 than men on trial 1 and did not up regulate baseline Hsp72 expression during trial 2. Thus, in response to an initial exercise challenge, women had a blunted cellular stress response compared to men. This sex difference may highlight the redundant mechanisms of estrogen and Hsp72 in mediating the stress response to exercise in the heat. Estrogen may help to stabilize cell membranes, thus reducing the need to up regulate Hsp72 after an acute stress. Thus, we reject our hypothesis that women would have an increased pre exercise expression of Hsp72, but retain our hypothesis that the post exercise response in women would be attenuated compared to men. In addition, there was no effect of menstrual phase either at rest or after exercise on Hsp72 expression. Therefore, we reject our hypothesis that during lut, when estrogen levels are elevated, there would be a decrease in Hsp72 at rest and after exercise compared to fol.

Hsp72 expression has a significant role in maintaining cellular homeostasis during and after stress $(4,26)$. While numerous animal studies have found sex differences in Hsp72 expression with acute hyperthermia or exercise, to our knowledge this is the first study to examine this effect in humans. Results from animal studies imply that estrogen is responsible for increased baseline Hsp72 expression $(14,15,23)$ and this up-regulation exerts protective effects that reduce the need for Hsp72 production during exercise $(16,19)$, ischemia $(17)$, or hyperthermic (18) stresses. In our study in humans, the baseline Hsp72 did not differ between sexes. However, men expressed greater Hsp72 compared to women in response to stress.
Thus, while it is understood that male animals increase Hsp72 to a greater extent in response to stress than females, we report for the first time that this sex effect is also evident in humans.

Acquired cellular thermotolerance occurs when a single exposure to a severe, but sub-lethal heat stress leads to protection against future, more severe heat exposure. This process involves the increased expression of basal Hsp72 $(2,3)$ and leads to decreased Hsp72 induction in response to a second exposure (24). In this fashion, Hsp72 can act as a marker for thermal history (10). In men, we found a greater percent increase in baseline PBMC Hsp72 content 12 days after trial 1 compared to women. Although this was not statistically significant, it is likely that the increased baseline expression seen in men in trial 2 abrogated the need for further Hsp72 production during trial 2. As such, neither men nor women showed a significant accumulation of Hsp72 in PBMCs during the second trial. Thus, it appears that Hsp72 may be regulated differently after an acute bout of exercise in the heat in non- heat acclimated men and women. It has not been previously appreciated that baseline Hsp72 could be up-regulated 12 days after a single acute bout of exercise in the heat. This data raises interesting questions regarding the role of thermotolerance in women. Estrogen may relegate the process of acquired cellular thermotolerance redundant, diminishing the need for Hsp72 accumulation.

This paper also is the first to examine possible effects of the menstrual cycle on Hsp72 expression. If estrogen is responsible for the decreased Hsp72 induction during exercise, then the variation of estrogen across the menstrual cycle could alter baseline or stress induced Hsp72 expression. The mechanism behind the relationship between estrogen and the blunted intracellular Hsp72 response to stress currently is not known. It was suggested that estrogen mediates this effect through a nongenomic hormonal pathway. Treating animals with tamoxifen, a known estrogen receptor agonist, caused the same blunted post exercise Hsp70 expression as in ovariectomized animals treated with $17 \beta$ and $17 \alpha$ estradiol (17). Since tamoxifen, $17 \beta$, and $17 \alpha$ estradiol all suppress the post exercise expression of Hsp70, researchers suggest that these estrogen related compounds stabilize cell membranes and attenuate oxidative stress (25). Such an effect could protect thermal sensitive cells against exercise-induced damage, and thereby result in a blunted Hsp72 expression. However, we found no difference in baseline or exercise Hsp72 response when women exercised in the fol compared to the lut phase. While our statistical power was limited with only 7 subjects included, there was no correlation between ovarian hormone concentrations and Hsp72 expression with $n=9$. Our findings are supported by animal data that suggest no effect of the estrous cycle on Hsp72 production in the pituitary or adrenal gland, spleen, lymph nodes, liver or heart in response to stress (27). Thus, the physiologic variations in estrogen and progesterone during the menstrual cycle may not be sufficient 
to alter Hsp72 expression.

Our main finding is that non-acclimated men increased Hsp72 more than women in PBMCs in response to exercise in the heat. After this single bout of exercise, men's Hsp72 was up-regulated for up to 12 days, suggesting that men had acquired cellular thermal tolerance. We suggest that estrogen may provide cellular protection and thus decrease the need to up-regulate Hsp72 in non-acclimated women. These data raise intriguing questions about the role of acquired cellular thermal tolerance between sexes.

\section{Acknowledgements}

None declared.

\section{Authors' Contribution}

Trevor Gillum developed the original idea and the protocol, abstracted and analyzed data, and wrote the manuscript. Matthew Kuennen, Cheryl Gourley, Karol Dokladny, Suzanne Schneider, and Pope Moseley contributed to the development of the protocol, data collection, abstracted data, and prepared the manuscript.

\section{Financial Disclosure}

There are no conflicts of interest with any author of this manuscript.

\section{Funding/Support}

This study was supported by NIH DHHS/HIH/NCRR 1UL1RR031977-01 General Clinical Research Center of the University of New Mexico, Gatorade Sports Science Institute, and UNM's Graduate Research and Development.

\section{Role of the Sponsor}

The funding organizations are public institutions and had no role in the design and conduct of the study; collection, management, and analysis of the data; or preparation, review, and approval of the manuscript. Funds were used to purchase lab supplies and equipment.

\section{References}

1. Moran DS, Eli-Berchoer L, Heled Y, Mendel L, Schocina M, Horowitz M. Heat intolerance: does gene transcription contribute? J Appl Physiol. 2006;100(4):1370-6.

2. Landry J, Bernier D, Chretien P, Nicole LM, Tanguay RM, Marceau N. Synthesis and degradation of heat shock proteins during development and decay of thermotolerance. Cancer Res. 1982;42(6):2457-61.

3. Li GC, Werb Z. Correlation between synthesis of heat shock proteins and development of thermotolerance in Chinese hamster fibroblasts. Proc Natl Acad Sci U S A. 1982;79(10):3218-22.

4. Hartl FU. Molecular chaperones in cellular protein folding. $\mathrm{Na}$ ture. 1996;381(6583):571-9.

5. Chen HW, Kuo HT, Wang SJ, Lu TS, Yang RC. In vivo heat shock protein assembles with septic liver NF-kappaB/I-kappaB complex regulating NF-kappaB activity. Shock. 2005;24(3):232-8.

6. Selkirk GA, McLellan TM, Wright HE, Rhind SG. Mild endotoxemia, NF-kappaB translocation, and cytokine increase during exertional heat stress in trained and untrained individuals. Am J
Physiol Regul Integr Comp Physiol. 2008;295(2):R611-23.

7. Selkirk GA, McLellan TM, Wright HE, Rhind SG. Expression of intracellular cytokines, HSP72, and apoptosis in monocyte subsets during exertional heat stress in trained and untrained individuals. Am J Physiol Regul Integr Comp Physiol. 20 09;296(3):R575-86.

8. Yoo CG, Lee S, Lee CT, Kim YW, Han SK, Shim YS. Anti-inflammatory effect of heat shock protein induction is related to stabilization of I kappa B alpha through preventing I kappa B kinase activation in respiratory epithelial cells. J Immunol. 2000;164(10):541623.

9. Fehrenbach E, Niess AM, Schlotz E, Passek F, Dickhuth HH, Northoff $H$. Transcriptional and translational regulation of heat shock proteins in leukocytes of endurance runners. J Appl Physiol. 2000;89(2):704-10.

10. Ryan AJ, Gisolfi CV, Moseley PL. Synthesis of 70K stress protein by human leukocytes: effect of exercise in the heat. J Appl Physiol. 1991;70(1):466-71.

11. Oishi Y, Taniguchi K, Matsumoto H, Ishihara A, Ohira Y, Roy RR. Muscle type-specific response of HSP60, HSP72, and HSC73 during recovery after elevation of muscle temperature. J Appl Physiol.2002;92(3):1097-103.

12. Campisi J, Leem TH, Greenwood BN, Hansen MK, Moraska A, Higgins $\mathrm{K}$, et al. Habitual physical activity facilitates stress-induced HSP72 induction in brain, peripheral, and immune tissues. Am J Physiol Regul Integr Comp Physiol. 2003;284(2):R520-30.

13. Flanagan SW, Ryan AJ, Gisolfi CV, Moseley PL. Tissue-specific HSP70 response in animals undergoing heat stress. Am J Physiol. 1995;268(1 Pt 2):R28-32.

14. Fekete A, Vannay A, Ver A, Rusai K, Muller V, Reusz G, et al. Sex differences in heat shock protein 72 expression and localization in rats following renal ischemia-reperfusion injury. Am J Physiol Renal Physiol. 2006;291(4):F806-11.

15. Voss MR, Stallone JN, Li M, Cornelussen RN, Knuefermann P, Knowlton AA. Gender differences in the expression of heat shock proteins: the effect of estrogen. Am J Physiol Heart Circ Physiol. 2003;285(2):H687-92.

16. Paroo Z, Dipchand ES, Noble EG. Estrogen attenuates postexercise HSP70 expression in skeletal muscle. Am J Physiol Cell Physiol. 2002;282(2):C245-51.

17. Paroo Z, Haist JV, Karmazyn M, Noble EG. Exercise improves postischemic cardiac function in males but not females: consequences of a novel sex-specific heat shock protein 70 response. Circ Res. 2002;90(8):911-7.

18. Shinohara T, Takahashi N, Ooie T, Ichinose M, Hara M, Yonemochi $\mathrm{H}$, et al. Estrogen inhibits hyperthermia-induced expression of heat-shock protein 72 and cardioprotection against ischemia/reperfusion injury in female rat heart. J Mol Cell Cardiol. 2004;37(5):1053-61.

19. McArdle A, Dillmann WH, Mestril R, Faulkner JA, Jackson MJ. Overexpression of HSP70 in mouse skeletal muscle protects against muscle damage and age-related muscle dysfunction. FASEB J. 2004;18(2):355-7.

20. Morton JP, Maclaren DP, Cable NT, Campbell IT, Evans L, Bongers $\mathrm{T}$, et al. Elevated core and muscle temperature to levels comparable to exercise do not increase heat shock protein content of skeletal muscle of physically active men. Acta Physiol (Oxf). 2007;190(4):319-27.

21. Taylor L, Midgley AW, Chrismas B, Madden LA, Vince RV, McNaughton LR. Daily quadratic trend in basal monocyte expressed HSP72 in healthy human subjects. Amino Acids. 2010;38(5):1483-8.

22. Brozek J, Grande F, Anderson JT, Keys A. Densitometric Analysis of Body Composition: Revision of Some Quantitative Assumptions. Ann N Y Acad Sci.1963;110:113-40.

23. Bombardier E, Vigna C, Iqbal S, Tiidus PM, Tupling AR. Effects of ovarian sex hormones and downhill running on fiber-type-specific HSP70 expression in rat soleus. J Appl Physiol. 2009;106(6):2009-15.

24. Radom-Aizik S, Zaldivar F, Jr, Leu SY, Cooper DM. A brief bout of exercise alters gene expression and distinct gene pathways in peripheral blood mononuclear cells of early- and late-pubertal females.J Appl Physiol. 2009;107(1):168-75.

25. Wiseman H, Quinn P, Halliwell B. Tamoxifen and related com- 


\section{Gillum Tet al.}

pounds decrease membrane fluidity in liposomes. Mechanism for the antioxidant action of tamoxifen and relevance to its anticancer and cardioprotective actions? FEBS Lett. 1993;330(1):536.

26. Welch WJ, Feramisco JR. Nuclear and nucleolar localization of the 72,000-dalton heat shock protein in heat-shocked mammalian cells. J Biol Chem. 1984;259(7):4501-13.

27. Nickerson M, Kennedy SL, Johnson JD, Fleshner M. Sexual dimorphism of the intracellular heat shock protein 72 response. Appl Physiol. 2006;101(2):566-75. 